\title{
Normal Dispersion Impact on OSNR in Optical Parametric Frequency Combs
}

\author{
C. K. Huynh, S. Zlatanovic \\ Advanced Photonic Technologies Branch \\ SPAWAR Systems Center Pacific \\ San Diego, USA \\ christopher.huynh@navy.mil
}

\author{
S. Radic \\ Dept. of Electrical and Computer Engineering \\ University of California San Diego \\ San Diego, USA
}

\begin{abstract}
We report on the improvement of OSNR in optical frequency combs generated by parametric mixing in optical fiber due to increasing normal dispersion and decreasing pulse width through numerical simulation and experimental validation.
\end{abstract}

Keywords—four-wave mixing; parametric processing

\section{INTRODUCTION}

Optical frequency combs have been utilized in replication of RF spectra to enable the parallelization of signal processing for applications such as staring wideband RF monitoring beyond the limitations set by a single backend digital receiver $[1,2]$. For such applications, optical signal-to-noise ratio (OSNR) is imperative to achieve high sensitivity and facilitate detection of low probability of detection/intercept signals [3].

The noise performance of optical frequency combs generated by parametric mixing has been investigated in the context of multicasting and noise figure [4]. This study has shown that finite normal dispersion can improve the overall noise figure of the parametric system from the theoretical limits set by a dispersion-free process [5]. The concept of localization of parametric mixing efficiency using normal dispersion inspired the search for the lowest achievable noise impact [6]. In this paper, we report on detailed preliminary findings on the impact of finite normal dispersion on the OSNR of a parametrically derived frequency comb.

\section{EXPERIMENTAL DESIGN \& RESULTS}

The OSNR impact on parametrically derived optical frequency combs by finite normal dispersion in highly nonlinear fiber was numerically investigated via an adaptive step nonlinear Schrödinger equation solver. The model implemented two simulated phase-locked semi-classical pumps exhibiting quantum-limited OSNR spaced at $50 \mathrm{GHz}$ and $200 \mathrm{GHz}$. The numerical model incrementally characterized the comb noise behavior in relation to chromatic dispersion and adimensionalized nonlinear length as defined by $\frac{1}{\gamma P L}$, where $\gamma$ is the nonlinear parameter, $\mathrm{P}$ is the pump power and $\mathrm{L}$ is the length of the fiber. The numerical results suggest that given normal dispersion of $-0.0 \sim-0.5 \mathrm{ps} / \mathrm{nm}-\mathrm{km}$, and a adimenaionalized nonlinear length of 1, appreciable OSNR improvement of about $2.5 \mathrm{~dB}$ can be seen in the case of $200 \mathrm{GHz}$ pump separation, whereas negligible OSNR improvement $(<1 \mathrm{~dB})$ can be seen in the case of $50 \mathrm{GHz}$ pump separation. This finding suggests that the pulse width is significant to the balance between dispersion and nonlinearity.

In the experimental architecture shown in Fig. 1, a pair of pumps seeded from a single master laser (M1) was injection locked and combined. Subsequently the pumps were phase dithered with a $20 \mathrm{dBm} 200 \mathrm{MHz} \mathrm{RF}$ tone to increase the Stimulated Brillouin Scattering (SBS) threshold to $32 \mathrm{dBm}$. The input power was attenuated after the EDFA to change the nonlinear length while maintaining constant OSNR performance of the pumps. In order to generate the optical frequency comb, the pumps were launched into a $250 \mathrm{~m}$ piece of dispersion-flattened highly nonlinear fiber (HNLF) with near-zero peak dispersion near the pump frequencies. Tension was employed to adjust the dispersion profile from

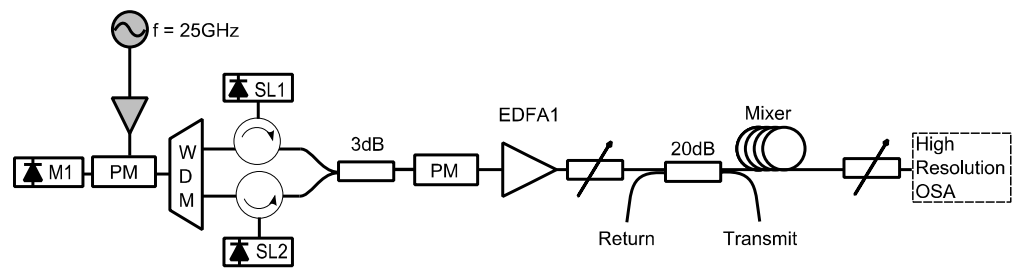

Fig. 1. The system design starts with a master laser (M1) at $193.5 \mathrm{THz}$ EO-modulated into a $25 \mathrm{GHz}$ pitched comb through two phase modulators (PM). Two pump seeds are narrowly filtered for injection locking lasers SL1 and SL2 at $193.475 \mathrm{THz}$ and $193.525 \mathrm{THz}$ for the $50 \mathrm{GHz}$ case and $193.4 \mathrm{THz}$ and 193.6 $\mathrm{THz}$ for the $200 \mathrm{GHz}$ case. The pumps were combined, and amplified in EDFA1. The EDFA output was attenuated to adjust the input power and control nonlinearity. A 4-port 99:1 directional coupler was used to monitor input power and return power. The mixer consisted of $250 \mathrm{~m}$ of dispersion flattened HNLF. The output of the mixer was attenuated and analyzed in a high resolution OSA. 


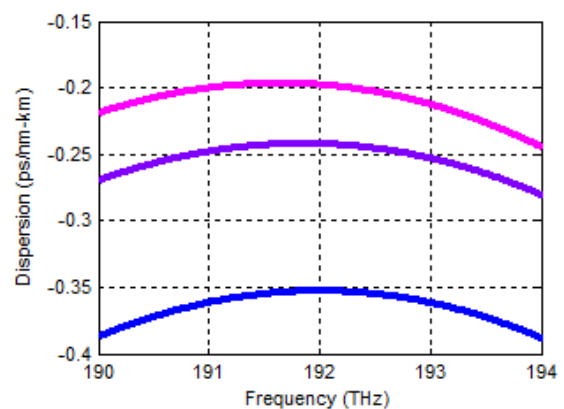

Fig. 2. The dispersion profiles of the tensioned dispersion-flattened highly nonlinear fiber. The peak dispersions were measured at -0.2 , -0.24 , and $-0.35 \mathrm{ps} / \mathrm{nm}-\mathrm{km}$. At $193.5 \mathrm{THz}$, the dispersion was measured at $-0.23,-0.27$, and $-0.37 \mathrm{ps} / \mathrm{nm}-\mathrm{km}$

$-0.2 \mathrm{ps} / \mathrm{nm}-\mathrm{km}$ to $-0.35 \mathrm{ps} / \mathrm{nm}-\mathrm{km}$ as shown in Fig. 2 .

In simulation, the OSNR improvement of individual comb tones by finite dispersion accumulated with longer mixer lengths. The most apparent improvement in experiment was found at maximum input power below the SBS condition as shown in Fig. 3 and Fig. 4. The total input power into the highly nonlinear fiber was $31.4 \mathrm{dBm}$ for both cases $50 \mathrm{GHz}$ and $200 \mathrm{GHz}$ pump spacing. Ten frequency modes are shown, which encompass the OSNR of each line in the parametrically generated comb. The combs are not wide when compared to previous work [7] due to the simplicity of the mixer design: a single piece of highly nonlinear fiber.

In Fig. 3, the OSNR of each mode was measured in the comb seeded by $50 \mathrm{GHz}$ separated pumps in $10 \mathrm{GHz}$ resolution bandwidth. This result shows $2 \mathrm{~dB}$ fluctuation in OSNR, but no overall improvement in OSNR. In Fig. 4, the comb seeded by $200 \mathrm{GHz}$ separated pumps exhibits overall OSNR improvement of approximately $10 \mathrm{~dB}$, and as much as $12 \mathrm{~dB}$ for two channels. Fig. 3 and Fig. 4 show a preliminary validation of the numerical finding that increasingly normal dispersion can improve the overall OSNR of a frequency comb generated by parametric mixing and that the shorter pulse in the $200 \mathrm{GHz}$ pump separation case would yield a larger improvement in OSNR.

The OSNR improvement in the $200 \mathrm{GHz}$ case is much larger than expected from simulations. This may be a result of the uncontrolled polarization states of the pumps launched into the parametric mixer. Tensioned induced birefringence and polarization mode dispersion may have influenced the result. This discrepancy from the numerical finding requires further investigation.

\section{CONCLUSION}

The impact of normal dispersion on OSNR in optical frequency combs generated by parametric mixing was shown. The results suggest that increasing amounts of normal dispersion have a positive impact on performance given a shorter pulse length.

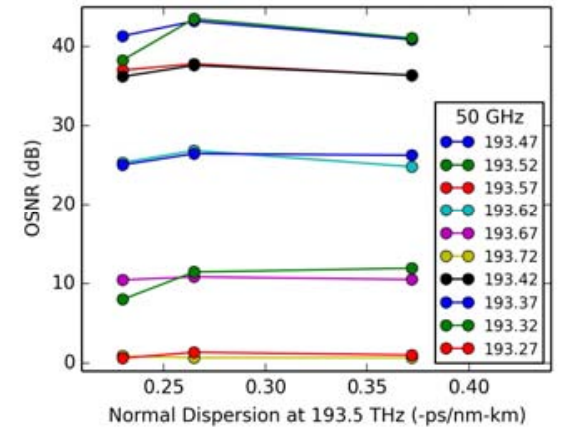

Fig. 3. The OSNR change across the parametric comb seeded by $50 \mathrm{GHz}$ spaced pumps with relation to increasing normal dispersion. RBW: $10 \mathrm{GHz}$.

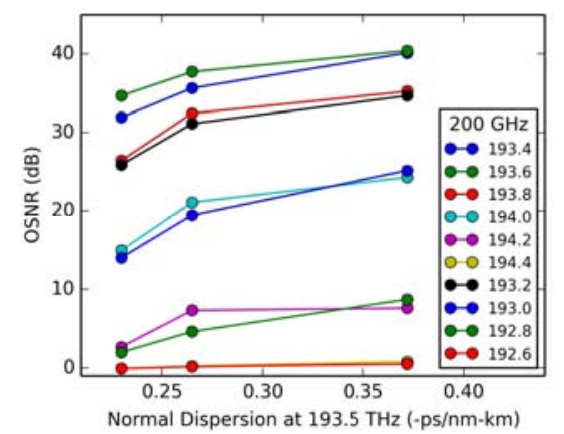

Fig. 4. The OSNR change across the parametric comb seeded by 200 $\mathrm{GHz}$ spaced pumps with relation to increasing normal dispersion. RBW: $10 \mathrm{GHz}$

\section{ACKNOWLEDGEMENTS}

The authors gratefully acknowledge the support from Office of Naval Research, and to Sumitomo Electric Industries for providing the highly-nonlinear fiber used in experiment.

\section{REFERENCES}

[1] Z. Li, et al. "A configurable Microwave Photonic Channelized Receiver Based on Dense Wavelength Division Multiplexing Using an Optical Comb." Optics Communications, 285, 9, pp. 2311-2315, 2012.

[2] X. Xie, et al. "Broadband Photonic RF Channelization Based on Coherent Optical Frequency Combs and I/Q Demodulators," Photonics Journal, IEEE, vol. 4, no. 4, pp. 1196-1202, Aug. 2012.

[3] C. Huynh, et al. "Wideband RF Analyzer with 32 Channels Based on Parametric Multicasting." Proc. IEEE Intl. Topical Meeting on Microwave Photonics (MWP), vol., pp.339,342, Oct. 2014.

[4] C. Huynh, et al. "Noise Performance of phase-insensitive multicasting in multi-stage parametric mixers," Opt. Express 21, 804-814 (2014).

[5] C. McKinstrie, et al. "Quantum noise properties of parametric amplifiers driven by two pump waves," Opt. Express 12, 5037-5066 (2004).

[6] Z. Tong, et al. "Generation of low-noise frequency replicas in parametric frequency combs via phase-sensitive process" Proc. SPIE 8964, Nonlinear Freq. Generation and Conversion: Materials, Devices, and Applications XIII, 89640M, Feb. 2014

[7] E. Myslivets, et al., "Generation of wideband frequency combs by continuouswave seeding of multistage mixers with synthesized dispersion,” Opt. Express 20, 3331-3344 (2012), 